\title{
Keeping the shape of plant tissue for visualizing metabolite features in segmentation and correlation analysis of imaging mass spectrometry in Asparagus officinalis
}

\author{
Ryo Nakabayashi ${ }^{1}$ (I) Kei Hashimoto ${ }^{1} \cdot$ Kiminori Toyooka $^{1} \cdot$ Kazuki Saito $^{1,2}$
}

Received: 23 September 2018 / Accepted: 2 February 2019 / Published online: 14 February 2019

(c) The Author(s) 2019

\begin{abstract}
Introduction Matrix-assisted laser desorption/ionization-imaging mass spectrometry (MALDI-IMS) is a powerful approach for visualizing the localization of metabolites.

Objectives A method to keep the shape of plant tissue needs to be developed for MALDI-IMS.

Methods The method was developed using transfer tape and double-sided conductive tape.

Results MALDI-IMS analysis using the developed method enabled to perform segmentation and correlation analysis of mass features.

Conclusion This proof-of-concept study showed that rutin localizes in the epidermis, developing tissue, and protoxylem in Asparagus officinalis.
\end{abstract}

Keywords Metabolomics $\cdot$ Imaging mass spectrometry $\cdot$ Liquid chromatography-tandem mass spectrometry $\cdot$ Specialized metabolite $\cdot$ Asparagus officinalis

\section{Introduction}

Specialized metabolites (previously called secondary metabolites) are significant natural products that are associated with certain species and accumulate in specific tissues and organs of plants. Previously, these metabolites were recognized as the byproducts of primary metabolites and were considered irrelevant (Hartmann 2007). However, recent phytochemical genomics studies have shown that they have important biological functions (Field et al. 2006; Massalha et al. 2017; Tohge et al. 2018). Comparative analysis of transcriptomics and metabolomics in transformants/mutants, which over-accumulate or lack certain metabolites by editing

Electronic supplementary material The online version of this article (https://doi.org/10.1007/s11306-019-1486-5) contains supplementary material, which is available to authorized users.

Ryo Nakabayashi

ryo.nakabayashi@riken.jp

1 RIKEN Center for Sustainable Resource Science, 1-7-22 Suehiro-cho, Tsurumi-ku, Yokohama 230-0045, Japan

2 Graduate School of Pharmaceutical Sciences, Chiba University, 1-8-1 Inohana, Chuo-ku, Chiba 260-8675, Japan biosynthetic genes, can be used for identifying the functions of metabolites in plants (Nakabayashi et al. 2014). To identify biosynthetic genes responsible for specialized metabolites, understanding the association of metabolite accumulation with gene expression at certain parts is important (Saito 2013). However, this step for identifying the localization of the metabolites is time consuming. Therefore, new approaches need to be developed to reduce the time required.

Matrix-assisted laser desorption/ionization-imaging mass spectrometry (MALDI-IMS) is a powerful approach for visualizing the localization of metabolites and is used in sections of organisms (Dong et al. 2016; Fujimura and Miura 2014; Lee et al. 2012; Sarabia et al. 2018; Sturtevant et al. 2016). Recently, this approach has been applied to identify the localization of specialized metabolites in plants (Enomoto et al. 2018; Jarvis et al. 2017; Li et al. 2014; Shiono et al. 2017). One of the major challenges of MALDI-IMS is to keep plant tissue shapes during the preparation of sections. Plant tissues contain large amounts of water. A freeze-dried process is necessary to remove the water content from sections placed on glass slides. This process results in peeling the section from the slide using typical methods. Overcoming this problem may improve regional analysis via MALDI-IMS. 
In the present study, a method named NakaMi was developed for the preparation of plant sections. As a proof of concept, this method was then applied to MALDI-IMS analysis on cross section in Asparagus officinalis (green asparagus), which is one of the staple crops in the world. A segmentation analysis showed that rutin (quercetin 3-O-rutinoside) localizes in the epidermis and developing stem tissue. Liquid chromatography-tandem mass spectrometry (LC-MS/ MS) using the authentic standard compound supported the MALDI-IMS analysis result. The IMS analysis suggested that rutin accumulates in the protoxylem of Asparagus. A correlation analysis showed that the ion derived from rutin was correlated with the mass features of other metabolites.

\section{Materials and methods}

\subsection{Chemicals}

The chemicals used in this study are listed in Supplementary Table 1.

\subsection{Plant materials}

Spears of Asparagus officinalis (green asparagus) were harvested at the end of April, 2018 from the Medicinal Plant Garden of Hoshi University, Japan.

\subsection{Preparing section}

To prepare sections for MALDI-IMS analysis, a fresh Asparagus spear was transversely cut (for an approximate thickness of $10 \mathrm{~mm}$ ) with a razor, then embedded with a reagent (Surgipath FSC22: Leica Microsystems, Germany), and frozen in a $-75{ }^{\circ} \mathrm{C}$ acetone bath (Histo-Tek Pino: Sakura Finetek Japan Co., Ltd., Tokyo, Japan). The frozen sample block was placed on a cryostat specimen disk and was cut with the knife blade until the desired tissue surface appeared. Transfer tape (Adhesive Tape Windows, Leica Microsystems, Germany) was placed on the face of the block to obtain sections (each with a thickness of $20 \mu \mathrm{m}$ ) in the CM3050S cryostat (Leica Microsystems, Germany). These sections were transferred to conductive $\mathrm{Cu}$ tape (double-sided, No. 796) (TERAOKA SEISAKUSHO, Co. Ltd.) on a glass slide (ITO coating, Bruker Daltonik $\mathrm{GmbH}$ ). The section on the glass slide was freeze-dried overnight at $-30{ }^{\circ} \mathrm{C}$ in the cryostat. For light microscopy, the frozen sections were stained with $0.05 \%$ toluidine-blue $\mathrm{O}$ solution for $1 \mathrm{~min}$ and washed with distilled water. The micrographs were acquired with a BX51 microscope equipped with a digital camera (Olympus cellSens and DP26).

\subsection{MALDI-IMS analysis}

A 2,5-dihydroxybenzoic acid (DHB) matrix solution (Supplementary Table 1) was sprayed on the prepared section that was on the glass slide using ImagePrep (Bruker Daltonik GmbH) running at the default parameters. The freeze-dried section with the matrix was analyzed in SolariX 7.0 T instrument (Bruker Daltonik $\mathrm{GmbH}$ ) for Fourier transform ion cyclotron resonance-mass spectrometry. The MALDI parameters are as follows: geometry, MTP 384 ground steel; plate offset, $100.0 \mathrm{~V}$; deflector plate, $200.0 \mathrm{~V}$; laser power, $50.0 \%$; laser shots, 200; frequency, $2000 \mathrm{~Hz}$; laser focus, small; raster width, $60 \mu \mathrm{m}$. The analytical conditions for the IMS analysis were identical to those for MALDI-MS analysis (Supplementary Methods).

The segmentation analysis was performed with the SCiLS Lab software (version 2019a). The mass features in the MALDI-IMS data (15,732 data points) were divided into seven groups by the bisecting $k$-means clustering (parameters: metric, Manhattan; minimal interval width, $\pm 1.8111 \mathrm{mDa}$ ). The three groups of ions (purple for the ground tissue, yellow for the vascular bundles, and green for the epidermis and developing tissue) are listed in Supplementary Data 1.

Pearson's correlation analysis was performed using the SCiLS Lab. The ion at $\mathrm{m} / \mathrm{z} 633.1425$ observed as $[\mathrm{M}+\mathrm{Na}]^{+}$from rutin was calculated with 3,243 mass features in half of the cross section (Supplementary Figure 1). A minimal interval width was set at $\pm 1.8111 \mathrm{mDa}$. Ions with correlation coefficient greater than 0.4 are listed in Supplementary Data 2.

\section{Results and discussion}

To determine which matrix reagent is appropriate for detecting wide range of metabolites, we first performed the screening of matrix reagents in 36 metabolites that belongs to 12 metabolite types (alkaloid, anthocyanidin, anthocyanin, flavonoid aglycone, flavonoid glycoside, glucosinolate, lignan, phenolamide/hydroxycinnamic acid amide, phenylpropanoid/coumarin, saponin/glycoalkaloid aglycone, saponin/glycoalkaloid, and sulfur-containing metabolite) using four matrix reagents, including $\alpha$-cyano-4-hydroxycinnamic acid (CHCA), 1,5-diaminonaphthalene, DHB, and 3-hydroxypicolinic acid, via MALDI-MS analysis (Supplementary Methods and Supplementary Table 2). Three compounds per metabolite type were selected, resulting in a total of 36 authentic standard compounds. The results suggest that CHCA and 
DHB are appropriate for detecting metabolites at a high sensitivity in positive ion mode of MALDI-IMS analysis. Notably, the signal intensities for DHB tended to be higher than those observed for CHCA. Negative ion mode that requires different parameters and reagents was out of scope in this study.

A method using transfer and conductive tape (termed the NakaMi method) was developed to keep the shape of the plant tissue on glass slides for MALDI-IMS analysis. All processes were performed in a chilled cryostat. The section was placed on the conductive tape (marketed product, not for MALDI-IMS). Subsequently, the transfer tape was gently removed by hand (Fig. 1). The plant tissues were not removed from the conductive tape after spraying on the matrix reagent and MALDI-IMS analysis.

For the IMS analysis, DHB was selected to detect different types of metabolites in Asparagus officinalis (green asparagus). To understand the pattern of mass features of detected metabolites, the segmentation analysis was performed using data from the cross section. The whole data comprised 15,732 data points; however, for data analysis, only half (7916 data points) were used for reducing the number of data points (Fig. 2). The colored map clearly showed that tissue-redundant metabolites existed in the section. The 11,365 mass features in three of the seven groups (green, yellow, and purple) subjected to segmentation analysis are provided in Supplementary Data 1.

Rutin is a health-promoting specialized metabolite with antioxidant activity (Butelli et al. 2008; Tohge et al. 2015). This metabolite accumulates in the epidermis of plants, e.g., in the leaves of Zea mays (Korte et al. 2015). In Asparagus, the localization of rutin has not been determined yet using MALDI-IMS. The IMS analysis showed that the ion at $m / z 633.1425$ was determined to be $[\mathrm{M}+\mathrm{Na}]^{+}$from rutin, which was distributed in the green group of the segmentation map. Light microscopy showed that the green represents
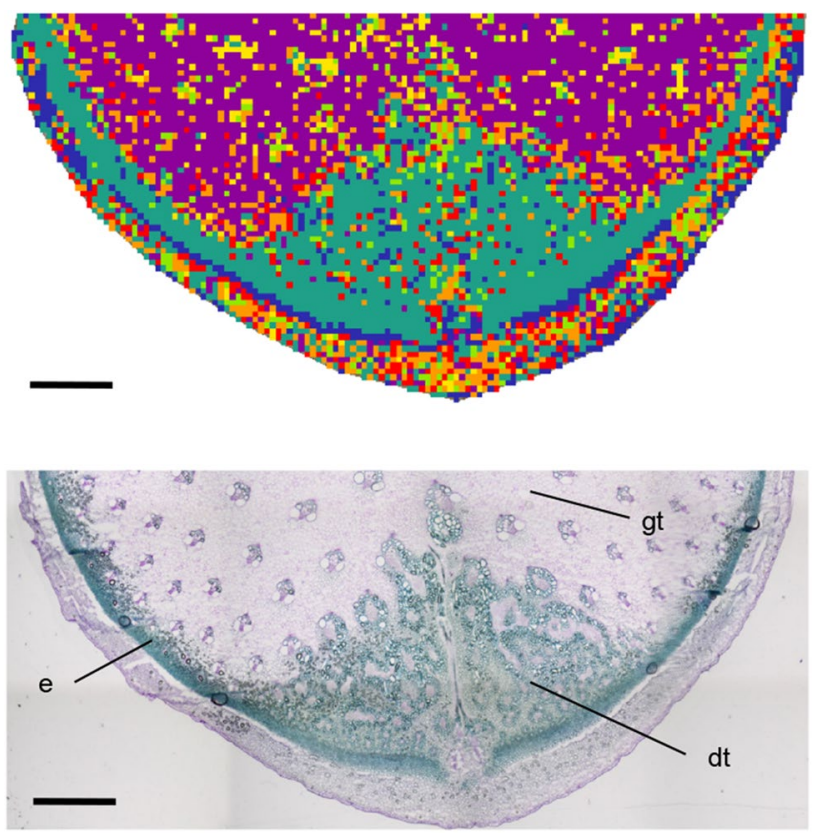

Fig. 2 Segmentation of detected mass features. Upper. The map highlighting the result in the segmentation analysis. All mass features were divided into seven colored groups (purple, red, orange, yellow, light green, blue, and green) in this case. Lower. Light microscopy in the sections stained using toluidine-blue O. $d t$ developing tissue, $e$ epidermis, gt ground tissue. Bar indicates $1 \mathrm{~mm}$

the epidermis and developing stem tissue (Figs. 2 and $3 a$ ). To identify rutin, LC-MS/MS analysis was performed using the authentic standard compound (Supplementary Figure 2 and Supplementary Methods), and it showed that the amount of rutin in the developing tissue and epidermis was more than that in the others' part including the ground tissue and vascular bundles (Fig. 3b). In addition, the IMS analysis showed that rutin accumulates in the protoxylem (Fig. 4). Previous research identified the localization of flavonol
A

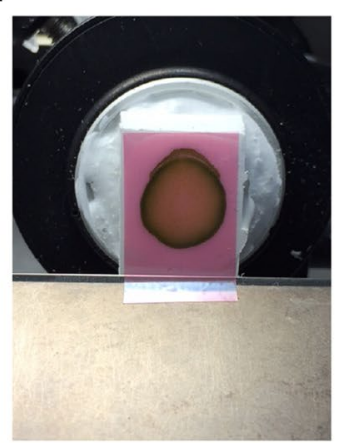

B

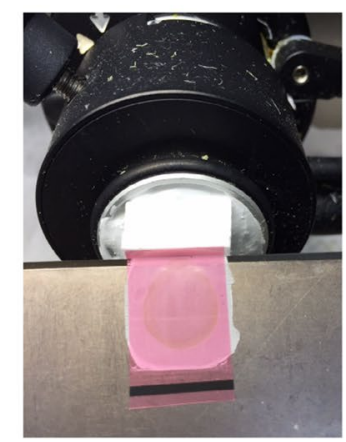

C

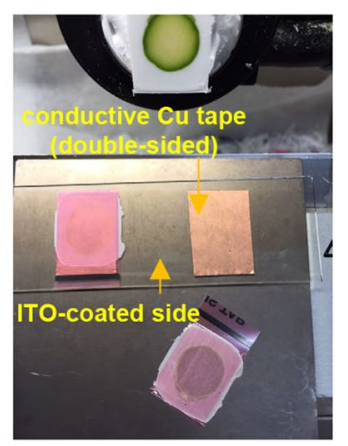

D

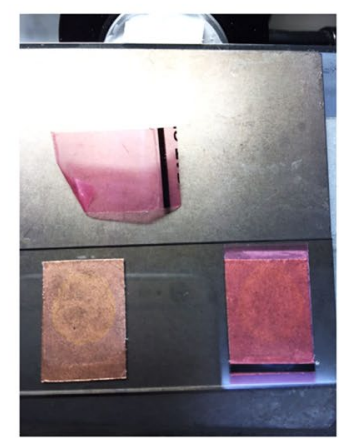

Fig. 1 Procedure of preparing plant sections for MALDI-IMS in this study. A The transfer tape (pink) was put on top of the block of the embedding reagent with the plant sample in the chilled cryostat. B
Sections were cut with the transfer tape. C The transfer tape was put on the conductive tape and dapped by finger. D The transfer tape was gently removed by hand 

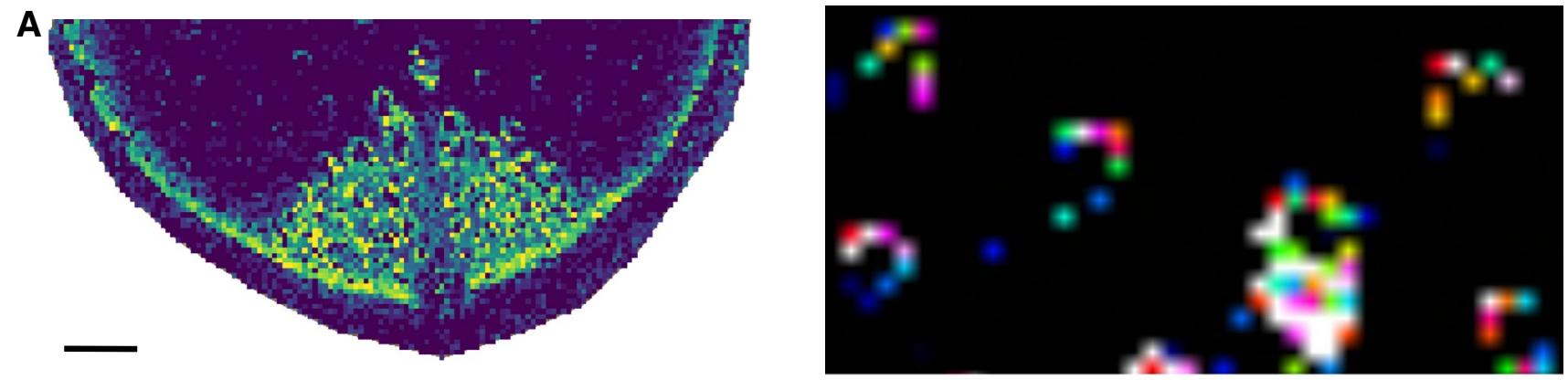

\section{B}

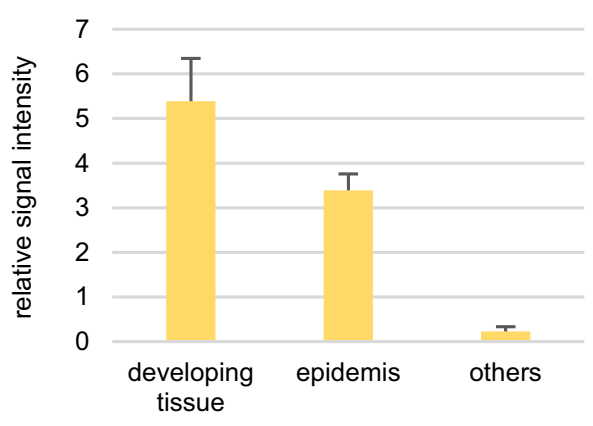

Fig. 3 Identifying the localization of rutin. A The localization of rutin was visualized with $\mathrm{m} / \mathrm{z}, 633.1425 \pm 1.8111$ as $[\mathrm{M}+\mathrm{Na}]^{+}$form on the cross section. Bar indicates $1 \mathrm{~mm}$. B Comparing the relative signal intensity of rutin in liquid chromatography-tandem mass spectrometry. The signal intensity of rutin was divided with that of the internal standard lidocaine in each sample. Others include the vascular and ground tissues. Bar indicates standard deviation $(n=3)$

monoglycosides, including quercetin-3-O- $\beta$-glucoside, in xylem parenchyma cells (Kasuga et al. 2008).

The localization of rutin in the epidermis in this study is a rational finding. Surprisingly, rutin accumulated in the developing tissue that was not exposed to sunlight yet. The following hypotheses for the function of rutin in developing tissue are considered: (1) rutin may provide the young cells of the stem an instantaneous mitigation of the production of reactive oxygen species (ROS) against its unavoidable future exposure to sunlight, due to its further outgrowth, (2) rutin may mitigate the production of ROS in the developing tissue (Manzano et al. 2014), or (3) xylem parenchyma cells accumulating rutin are differentiated in the developing tissue. To confirm these hypotheses, further studies need to be performed. For instance, correlation analysis is a powerful tool to understand co-localized metabolites in the protoxylem. Data from the region of the ground tissue and vascular bundles were used for the analysis (Supplementary Figure 1). This showed that the observed ion at $m / z 633.1425$ was correlated with 177 mass features (Pearson correlation coefficient PCC, $\geq 0.4$ ) (Supplementary Data 2). The correlation coefficient of monoisotopic ion $(\mathrm{m} / \mathrm{z}$ 633.1425) to its isotopic ion including ${ }^{13} \mathrm{C}(\mathrm{m} / \mathrm{z} 634.1455)$ was 0.41 . The IMS data

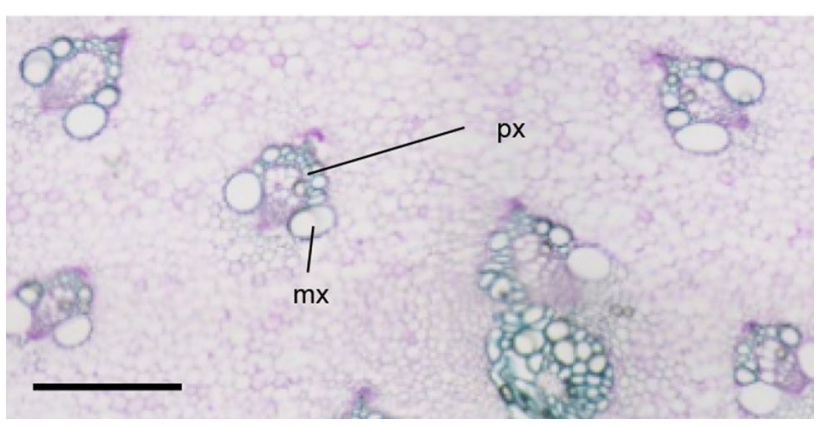

Fig. 4 The localization of rutin in the protoxylem characterized by the IMS analysis. Upper. The localization of rutin with the ion $\mathrm{m} / \mathrm{z}$ $633.1425 \pm 1.8111$ as $[\mathrm{M}+\mathrm{Na}]^{+}$. Lower. Light microscopy in the sections stained using toluidine-blue O. $m x$ metaxylem, $p x$ protoxylem. Bar indicates $500 \mu \mathrm{m}$

included data points in which both the monoisotopic and isotopic ion were detected in higher signal intensity, and only monoisotopic ion was detected in lower signal intensity. The bias to detecting only the monoisotopic ion resulted in the lower value. Based on this result, the highly correlated ions with the ion at $m / z 633.1425$ can be candidates for chemical assignment in the future in another study.

Identification of the metabolites may help to understand the function of rutin in the protoxylem. Integrated metabolomics with transcriptomics in the protoxylem can provide the associations of genes with rutin. The function of rutin may be predicted using annotations of genes associated with the metabolites. It is known that Asparagus is a transformable plant (Bytebier et al. 1987). Comparative analysis of the wild type gene and a transformant that lacks rutin can help directly understand its function.

The chemical diversity of the metabolome in the plant kingdom is vast. To probe the metabolome, polyhedral approaches are required. For example, some metabolites have been specifically detected via either MALDI-MS or electrospray ionization-MS (Nakabayashi et al. 2017). Additionally, analysis of these types of metabolites via IMS is becoming more common in the plant science field (Boughton et al. 2016; Etalo et al. 2015). The combination of both approaches is necessary for the comprehensive analysis of the localization of such metabolites. 
Acknowledgements We thank Jin Nakashima (Noble Foundation) and Daisuke Miura (National Institute of Advanced Industrial Science and Technology) for discussions regarding the NakaMi method, Mikiko Koizumi and Tetsuya Mori (RIKEN CSRS) for assisting with the MALDI-MS and LC-MS/MS analyses, Hiroshi Sudo (Hoshi University) for providing the Asparagus spears, and Jonathan Gershenzon (Max Planck Institute) and Hiromitsu Takayama (Chiba University) for providing authentic standard compounds. We also thank Takashi Nirasawa (Bruker Japan K. K.) and Amit Rai (Chiba University) for useful comments on the manuscript. This work was partially supported by the Science and Technology Research Promotion Program for Agriculture, Forestry, Fisheries and Food Industry (Ministry of Agriculture, Fisheries and Food), and the Japan Advanced Plant Science Network.

Author contributions RN conceived and designed the study. RN, KH, $\mathrm{KT}$ prepared the samples and collected the data. RN and KS wrote the manuscript and $\mathrm{RN}, \mathrm{KH}, \mathrm{KT}$, and KS discussed this results. KS supervised this project.

\section{Compliance with ethical standards}

Conflict of interest The authors declare no conflicts of interest.

Ethical approval This research does not involve human participants and/or animals.

Informed consent Informed consent was obtained from all participants.

Open Access This article is distributed under the terms of the Creative Commons Attribution 4.0 International License (http://creativeco mmons.org/licenses/by/4.0/), which permits unrestricted use, distribution, and reproduction in any medium, provided you give appropriate credit to the original author(s) and the source, provide a link to the Creative Commons license, and indicate if changes were made.

\section{References}

Boughton, B. A., Thinagaran, D., Sarabia, D., Bacic, A., \& Roessner, U. (2016). Mass spectrometry imaging for plant biology: A review. Phytochemistry Reviews, 15, 445-488.

Butelli, E., et al. (2008). Enrichment of tomato fruit with health-promoting anthocyanins by expression of select transcription factors. Nature Biotechnology, 26, 1301-1308.

Bytebier, B., Deboeck, F., Degreve, H., Vanmontagu, M., \& Hernalsteens, J. P. (1987). T-DNA organization in tumor cultures and transgenic plants of the monocotyledon Asparagus officinalis. Proceedings of the National Academy of Sciences of the United States of America, 84, 5345-5349.

Dong, Y. H., Li, B., \& Aharoni, A. (2016). More than pictures: When MS imaging meets histology. Trends in Plant Science, 21, 686-698.

Enomoto, H., Sato, K., Miyamoto, K., Ohtsuka, A., \& Yamane, H. (2018). Distribution analysis of anthocyanins, sugars, and organic acids in strawberry fruits using matrix-assisted laser desorption/ ionization-imaging mass spectrometry. Journal of Agricultural and Food Chemistry, 66, 4958-4965.

Etalo, D. W., De Vos, R. C. H., Joosten, M. H. A. J., \& Hall, R. D. (2015). Spatially resolved plant metabolomics: Some potentials and limitations of laser-ablation electrospray ionization mass spectrometry metabolite imaging. Plant Physiology, 169, 1424-1435.

Field, B., Jordan, F., \& Osbourn, A. (2006). First encounters-deployment of defence-related natural products by plants. New Phytologist, 172, 193-207.
Fujimura, Y., \& Miura, D. (2014). MALDI mass spectrometry imaging for visualizing in situ metabolism of endogenous metabolites and dietary phytochemicals. Metabolites, 4, 319-346.

Hartmann, T. (2007). From waste products to ecochemicals: Fifty years research of plant secondary metabolism. Phytochemistry, 68, 2831-2846.

Jarvis, D. E., et al. (2017). The genome of Chenopodium quinoa. Nature, 542, 307-312.

Kasuga, J., Hashidoko, Y., Nishioka, A., Yoshiba, M., Arakawa, K., \& Fujikawa, S. (2008). Deep supercooling xylem parenchyma cells of katsura tree (Cercidiphyllum japonicum) contain flavonol glycosides exhibiting high anti-ice nucleation activity. Plant Cell and Environment, 31, 1335-1348.

Korte, A. R., Yandeau-Nelson, M. D., Nikolau, B. J., \& Lee, Y. J. (2015). Subcellular-level resolution MALDI-MS imaging of maize leaf metabolites by MALDI-linear ion trap-Orbitrap mass spectrometer. Analytical and Bioanalytical Chemistry, 407, 2301-2309.

Lee, Y. J., Perdian, D. C., Song, Z. H., Yeung, E. S., \& Nikolau, B. J. (2012). Use of mass spectrometry for imaging metabolites in plants. The Plant Journal, 70, 81-95.

Li, B., Bhandari, D. R., Janfelt, C., Rompp, A., \& Spengler, B. (2014). Natural products in Glycyrrhiza glabra (licorice) rhizome imaged at the cellular level by atmospheric pressure matrix-assisted laser desorption/ionization tandem mass spectrometry imaging. The Plant Journal, 80, 161-171.

Manzano, C., et al. (2014). The emerging role of reactive oxygen species signaling during lateral root development. Plant Physiology, $165,1105-1119$.

Massalha, H., Korenblum, E., Tholl, D., \& Aharoni, A. (2017). Small molecules below-ground: The role of specialized metabolites in the rhizosphere. The Plant Journal, 90, 788-807.

Nakabayashi, R., et al. (2014). Enhancement of oxidative and drought tolerance in Arabidopsis by overaccumulation of antioxidant flavonoids. The Plant Journal, 77, 367-379.

Nakabayashi, R., Hashimoto, K., Toyooka, K., \& Saito, K. (2017). Topdown metabolomic approaches for nitrogen-containing metabolites. Analytical Chemistry, 89, 2698-2703.

Saito, K. (2013). Phytochemical genomics-A new trend. Current Opinion in Plant Biology, 16, 373-380.

Sarabia, L. D., et al. (2018). High-mass-resolution MALDI mass spectrometry imaging reveals detailed spatial distribution of metabolites and lipids in roots of barley seedlings in response to salinity stress. Metabolomics, 14, 63.

Shiono, K., et al. (2017). Multi-imaging of cytokinin and abscisic acid on the roots of rice (Oryza sativa) using matrix-assisted laser desorption/lonization mass spectrometry. Journal of Agricultural and Food Chemistry, 65, 7624-7628.

Sturtevant, D., Lee, Y. J., \& Chapman, K. D. (2016). Matrix assisted laser desorption/ionization-mass spectrometry imaging (MALDIMSI) for direct visualization of plant metabolites in situ. Current Opinion in Biotechnology, 37, 53-60.

Tohge, T., et al. (2015). Ectopic expression of snapdragon transcription factors facilitates the identification of genes encoding enzymes of anthocyanin decoration in tomato. The Plant Journal, 83, 686-704.

Tohge, T., de Souza, L. P., \& Fernie, A. R. (2018). On the natural diversity of phenylacylated-flavonoid and their in planta function under conditions of stress. Phytochemistry Reviews, 17, 279-290.

Publisher's Note Springer Nature remains neutral with regard to jurisdictional claims in published maps and institutional affiliations. 